\title{
A UK and Irish survey of enteral nutrition practices in paediatric intensive care units
}

\author{
Lyvonne Tume ${ }^{1,2 *}$, Bernie Carter ${ }^{1,3}$ and Lynne Latten ${ }^{1}$ \\ ${ }^{1}$ Alder Hey Children's NHS Foundation Trust, Eaton Road, Liverpool L12 2AP, UK \\ ${ }^{2}$ Liverpool John Moores University, Liverpool, UK \\ ${ }^{3}$ The University of Central Lancashire, Preston, Lancashire, UK
}

(Submitted 8 February 2012 - Final revision received 31 May 2012 - Accepted 31 May 2012 - First published online 1 August 2012)

\begin{abstract}
The aim of the present study was to describe the present knowledge of healthcare professionals and the practices surrounding enteral feeding in the UK and Irish paediatric intensive care unit (PICU) and propose recommendations for practice and research. A cross-sectional (thirty-four item) survey was sent to all PICU listed in the Paediatric Intensive Care Audit Network (PICANET) database (http://www. picanet.org.uk) in November 2010. The overall PICU response rate was 90\% (27/30 PICU; 108 individual responses in total). The overall breakdown of the professional groups was 59\% nursing staff (most were children's nurses), $27 \%$ medical staff, $13 \%$ dietitians and $1 \%$ physician assistants. Most units (96\%) had some written guidance (although brief and generic) on enteral nutrition (EN); $85 \%$ of staff, across all professional groups $(P=0.672)$, thought that guidelines helped to improve energy delivery in the PICU. Factors contributing to reduced energy delivery included: fluid-restrictive policies (60\%), the child just being 'too ill' to feed (17\%), surgical post-operative orders (16\%), nursing staff being too slow in starting feeds (7\%), frequent procedures requiring fasting (7\%) and haemodynamic instability (7\%). What constituted an 'acceptable' level of gastric residual volume (GRV) varied markedly across respondents, but GRV featured prominently in the decision to both stop EN and to determine feed tolerance and was similar for all professional groups. There was considerable variation across respondents about which procedures required fasting and the duration of this fasting. The present survey has highlighted the variability of the present enteral feeding practices across the UK and Ireland, particularly with regard to the use of GRV and fasting for procedures. The present study highlights a number of recommendations for both practice and research.
\end{abstract}

Key words: Paediatric nutrition: Intensive care: Enteral feeding: Surveys

Optimal nutrition in paediatric intensive care (PIC) plays an important role in improving patient outcomes through sustaining organ function and preventing dysfunction of the cardiovascular, respiratory and immune systems ${ }^{(1,2)}$. Enteral nutrition (EN) is preferential to parenteral nutrition in critically ill patients for reasons including maintaining gut integrity and reducing the risk of infection ${ }^{(3)}$. A recent guideline found that EN in PIC was interrupted in nearly one-third of patients due to intolerance to feeds (high gastric residual volume (GRV), emesis or diarrhoea), blocked/misplaced feeding tubes or medical procedures requiring fasting ${ }^{(4)}$. Many of these interruptions were avoidable and impacted on patient outcomes ${ }^{(4)}$. Other factors that impact on $\mathrm{EN}$ include fluid restriction and feed intolerance related to haemodynamic instability and inappropriate feed stoppage due to poor adherence to guidelines ${ }^{(5,6)}$. It was decided, therefore, to describe the present knowledge of healthcare professionals and the practices surrounding enteral feeding in the UK and Irish PIC units (PICU) and propose recommendations for practice and research.

\section{Methods}

The present cross-sectional (thirty-four item, predominantly close-ended) survey was developed to describe the present knowledge of healthcare professionals and the practices surrounding enteral feeding in the UK and Irish PICU (see Appendix). The Paediatric Intensive Care Society Study Group's (PICS-SG) group lead was approached to determine if ethical approval was required, but they determined that it was not required for the present study. The study was approved by the PICS-SG and registered as an audit with the National Health Service (NHS) Trust.

Abbreviations: EN, enteral nutrition; GRV, gastric residual volume; ICU, intensive care unit; NEC, necrotising enterocolitis; PIC, paediatric intensive care; PICS-SG, Paediatric Intensive Care Society Study Group; PICU, paediatric intensive care unit. 
No suitable and validated pre-existing tool was identified; hence, the survey team (the three authors and a consultant nurse (Andy Darbyshire) from a PICU) developed the survey using an iterative process of question development and refinement. The intention of the present survey was not to develop a fully validated tool, but to create a tool with sufficient robustness. The processes undertaken aimed to provide a level of face and content validity. The tool was initially developed as a paper-based pilot tool, with input and review from the Trust's Research and Review Committee; this thirty-sevenitem tool was then piloted in a single centre with 118 staff (64\% response rate). Following this pilot study, three questions were removed, the method of determining energy requirements (as so few medical or nursing staff knew this), a question about laxative use that was problematic and a question about dietetic referral, as these were not felt to specifically address the study aims.

The final version was then transferred across to an electronic format to be sent out nationally. The question domains and specific questions were built on an extensive review of the literature and experiential knowledge of the practice. The survey was designed to be user friendly, unambiguous and to minimise the time burden for completion. This meant that careful decisions were taken about the breadth and depth of the survey, resulting in some potential valuable domains not being addressed (e.g. dietitians' workload in PICU and specific prescribing practices). The survey was designed using single response answers, multiple response answers, ranked answers and free text, as appropriate to specific questions. Careful instructions about how to complete the survey were provided. This cross-sectional, thirty-four-item electronic survey (on SurveyMonkey ${ }^{\circledR}$; www.surveymonkey.com) was sent out to all PICU listed in the Paediatric Intensive Care Audit Network (PICANET) database (http://www.picanet.org.uk). The link to this e-survey was emailed to all lead consultants, lead nurses and all members of the PICS-SG in November 2010 and asked to forward this survey link to up to ten members (various disciplines and experience) of their team.

The acceptable unit response rate was set at $70 \%$. Two reminders were sent if a unit had not responded. As the study was exploratory, most results were analysed descriptively and involved the differences in enteral feeding practices across the PICU. Inferential data analysis was undertaken in SPSS v15 (SPSS, Inc.) by L. T. and examined; wherever possible, the difference between nurses', doctors' and dietitian's views of enteral feeding were compared using the $\chi^{2}$ test (a $P$ value $<0.05$ was considered significant). Most results are presented by individual responses (as per the aim of the survey), but where appropriate, unit responses are presented. Percentages do not always add up to $100 \%$ (e.g. where the staff members were asked to identify the top three factors').

\section{Results}

The overall PICU response rate from the e-survey was $90 \%$ (27/30 PICU; 108 individual responses, 1-21 responses per unit, mean unit response rate 3 ).

\section{Demographics of the respondents}

Of the PICU staff responding to the survey, $41 \%(11 / 27)$ were from combined cardiac and general PICU, 48\% (13/27) were from general PICU, 7\% (2/27) were from cardiac intensive care units (ICU) and 5\% (1/27) from other specialist ICU. There was a cross-section of respondents (Table 1; 69\% (74/108) had over 5 years PIC experience). The overall breakdown of the professional groups was: 59\% ( $n$ 64) nursing staff (most were children's nurses); $27 \%$ ( $n$ 29) medical staff; $13 \%$ ( $n$ 14) dietitians; and 1\% ( $n$ 1) physician assistants.

\section{Feeding on paediatric intensive care units}

Most units $(96 \% ; 26 / 27)$ had some written guidance (although brief and generic) on EN; and 85\% (88/103) of staff, across all professional groups ( $P=0.672$ ), thought that guidelines helped to improve energy delivery in the PICU. There was a perception by respondents that two groups of critically ill children fared worst in terms of energy delivery; these were children with cardiac conditions (77.6\%) and children after abdominal surgery $(61.5 \%)$. A number of contraindications to enteral feeding in PIC were cited with suspected necrotising enterocolitis (NEC), the most common contraindication to EN ( $88 \%, 91 / 104)$, followed by post-operative abdominal surgery (46\%, 48/104), high serum lactates $(11 \%, 11 / 104)$ and postoperative coarctation of the aorta $(11 \%, 11 / 104)$ (Fig. 1). In terms of assessing weight gain in critically ill children, less than one-half of respondents (29.9\%) said their unit had a policy for how often children were weighed, and one-third of respondents $(37.5 \%)$ said this was weekly, with $31.3 \%$ saying twice weekly. However, when asked when they thought children in a PICU should be weighed, over onehalf $(51.9 \%)$ said when the child had been on the PICU for more than 1 week.

When asked about how much of the child's prescribed energy intake they actually received, 70\% (75/107) of the staff stated that, on average, they thought children got less than $60 \%$ of their prescribed energy intake and $30 \%$ $(32 / 107)$ of them stated they received more than $60 \%$ of their required energy. This did not differ by professional

Table 1. Breakdown of respondents ( $n$ 108)

(Number of respondents and percentages)

\begin{tabular}{llc}
\hline & \multicolumn{2}{c}{ Respondents } \\
\cline { 2 - 3 } Professional & $n$ & $\%$ \\
\hline PIC consultant & 19 & $17 \cdot 6$ \\
Consultant anaesthetist & 2 & $1 \cdot 8$ \\
PIC registrars & 7 & $6 \cdot 5$ \\
Senior house officer & 1 & $0 \cdot 9$ \\
Dietitian & 14 & 13 \\
Charge nurses/sister/ward manager & 24 & $22 \cdot 2$ \\
Nurse educator & 6 & $5 \cdot 6$ \\
Research nurse & 3 & $2 \cdot 8$ \\
Advanced nurse practitioner & 4 & $3 \cdot 7$ \\
Physician assistant & 1 & $0 \cdot 9$ \\
Staff nurses (ICU trained) & 21 & $19 \cdot 4$ \\
Staff nurses (non-ICU trained) & 6 & $5 \cdot 6$
\end{tabular}

PIC, paediatric intensive care; ICU, intensive care unit. 


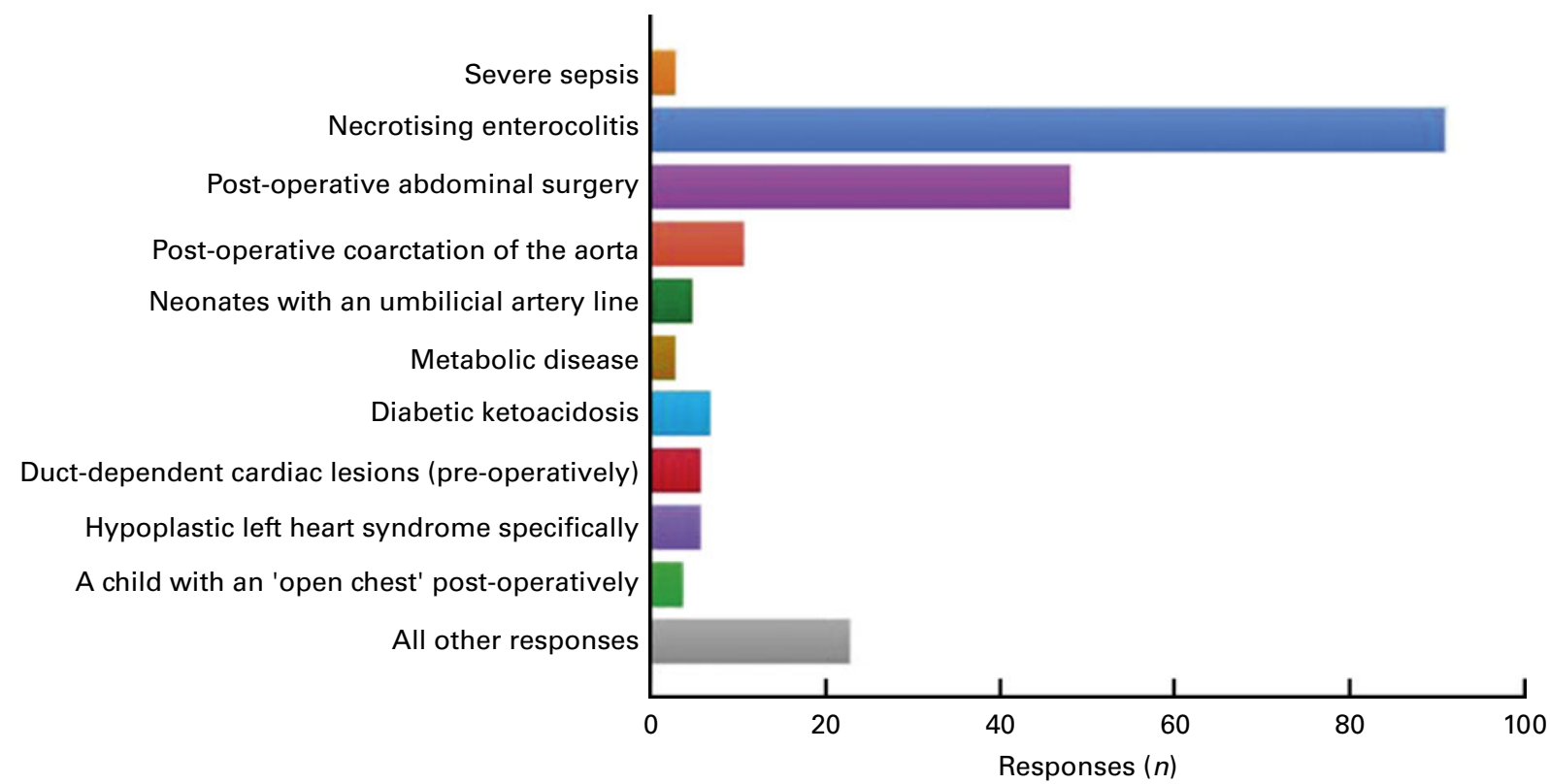

Fig. 1. Perceived contraindications for enteral feeding, in response to the question 'Which of the following conditions do you think are absolute contraindications to enteral feeding?'. (A colour version of this figure can be found online at http://www.journals.cambridge.org/bjn).

group $(P=0 \cdot 489)$. Fluid-restrictive policies $(60 \%)$, the child just being 'too ill' to feed (17\%), surgical post-operative orders $(16 \%)$, nursing staff being too slow in starting feeds (7\%), frequent procedures requiring fasting (7\%) and haemodynamic instability $(7 \%)$ were key factors identified in poor energy delivery. In terms of starting and stopping enteral feeds, $45 \%(45 / 101)$ of the respondents said there was 'no target start time' for enteral feeding; 25\% (24/101) stated 'just when the child was stable enough' (see Fig. 2); and $24 \%(24 / 101)$ of the respondents stated their guidelines were to start feeds within $4-6 \mathrm{~h}$ of admission. Across all professional groups $(P=0.615)$, the highest ranking reason to stop EN was when NEC was suspected $(66 \%, 57 / 87)$, followed by high GRV (32\%, 19/60) or gastrointestinal bleeding (29\%, 10/35). The top three signs used to determine feed tolerance were the amount of GRV, the absence of vomiting, followed by no abdominal distension and bowel sounds. All three professional groups placed a similar level of importance on GRV as an indicator to stop feeds $(P=0 \cdot 173)$

\section{Gastric residual volumes}

What constituted an 'acceptable' level of GRV varied markedly (50 different and subjective responses) ranging from 3 (47\%) to $10 \mathrm{ml} / \mathrm{kg}(11 \%)$ for a $5 \mathrm{~kg}$ infant. Broadly, it was felt that GRV had to be calculated by a percentage of what had been fed (range 25-100\%) or how many hours worth of feed remained (range 3 to $>6 \mathrm{~h}$ worth of feed) or an amount in $\mathrm{ml} / \mathrm{kg}$ (responses ranged from 4 to $5 \mathrm{ml} / \mathrm{kg}$ over a $4-\mathrm{h}$ period). There were no significant differences between professional groups $(P=0.903)$. The differences were more pronounced for an acceptable GRV in a $50 \mathrm{~kg}$ adolescent (range $100 \mathrm{ml}(1 \% ; 0.5 \mathrm{ml} / \mathrm{kg})$ to $400 \mathrm{ml}(12 \% ; 8 \mathrm{ml} / \mathrm{kg})$ ). For an adolescent, the majority expressed the acceptable level as

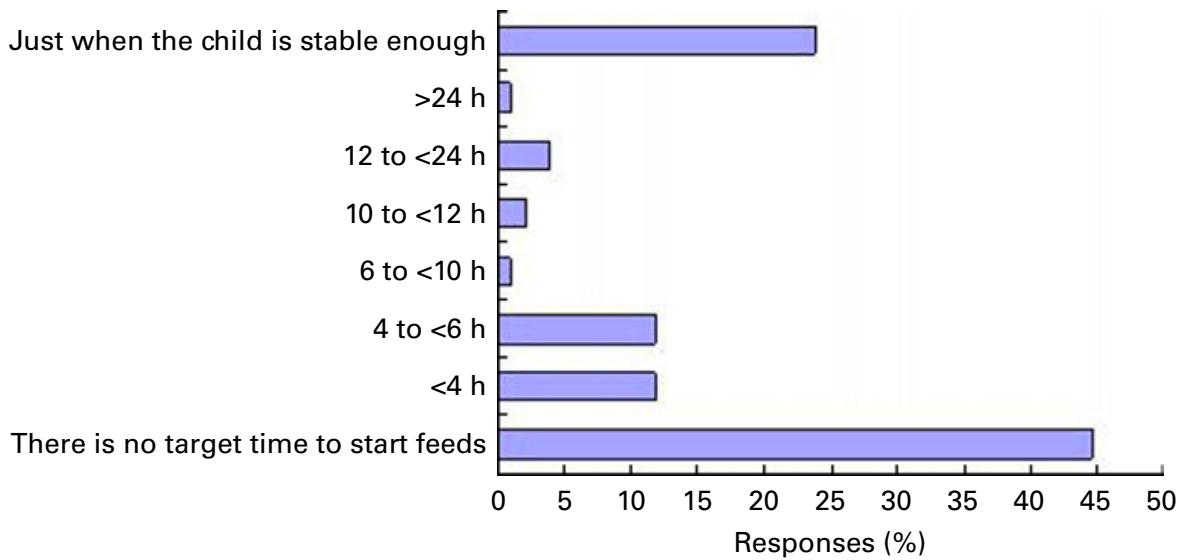

Fig. 2. Starting times for enteral feeds in the pediatric intensive care unit (PICU), in response to the question 'Does your unit have a target time for starting enteral feeds after PICU admission? and if so what is this?'. (A colour version of this figure can be found online at http://www.journals.cambridge.org/bjn). 
'percentage of feed given' (range $>25$ to $>70 \%$ of feed). There were no differences between professional groups $(P=0 \cdot 174)$. In terms of patient-related factors that affect GRV, $63 \%(54 / 86)$ of respondents said the site of the feeding tube was 'most important', followed by whether continuous feeds were used and the amount of gastric juice the patient produced. In terms of technical factors, $66 \%$ (52/79) said that the position of the feeding tube would be the most important factor affecting aspirate volume obtained. Migration of the feeding tube and other factors such as syringe size, type of feeding tube and nurse's technique were rated less important in affecting the amount of aspirate obtained. For both technical- and patient-related factors, nurses placed significantly more importance on the site of the feeding tube than did doctors or dietitians $(P=0 \cdot 021)$.

\section{Improving feed tolerance and fasting for procedures}

The first action that the respondents $(77 \%, 48 / 52)$ would use to improve feed tolerance would be to stop the feeds for a while and re-check the aspirate, followed by changing the continuous feeds and then starting a pro-kinetic agent. There was no difference between professional groups ( $P=0.610)$. A total of $42 \%(24 / 57)$ of the respondents stated that they would change from bolus to continuous feeds if the child was very ill, 32\% (30/93) stated that their standard regimen used continuous feeds. Significantly more nurses would consider changing to continuous feeds $(P=0 \cdot 019)$ if the child was not tolerating bolus feeds. Most respondents ( $82 \%$ ) said they always or sometimes used pro-kinetic agents. In all, $86 \%(87 / 101)$ stated that they used trophic feeds, although each provided a different response about what constituted trophic feeds. The most common definition of trophic feed was between 2 and $15 \mathrm{ml} / \mathrm{kg}$ every $1-3 \mathrm{~h}$ or $2-10 \mathrm{ml} / \mathrm{h}$. When asked about how early would parenteral nutrition be considered after feed intolerance, $34 \%$ of respondents stated between 48 and $72 \mathrm{~h} ; 33 \%$ said between 24 and $48 \mathrm{~h}$; $19 \%$ saying more than $72 \mathrm{~h} ; 2 \%$ stating $<24 \mathrm{~h}$; and $11 \%$ did not know. Although we did not specifically ask about naso-jejunal (or post-pyloric) feeding, a number of answers alluded to considering this, with a clinician from one unit claiming it was their default method to feed enterally. Fasting children for procedures on PIC was a significant problem and there was considerable variation across respondents about which procedures required fasting (Fig. 3) and the duration of fasting required (mean fasting time, Fig. 4). All staff fasted children for extubation and for theatre.

\section{Discussion}

To our knowledge, there have been no previous surveys of the UK and Irish paediatric ICU practices and staff views on enteral feeding. Previous surveys have primarily focused on adult intensive care nurses and found that practices regarding management of enteral feeding varied widely among nurses and that nursing practices alone may be contributing to underfeeding in critically ill patients ${ }^{(7-10)}$. Four prospective cohort studies show that guidelines help improve energy delivery in the PICU ${ }^{(2,6,11)}$. Most PICU had some written guidance on EN and most respondents (85\%) believed that guidelines helped improve energy delivery in the PICU. Most staff perceived that, on average, children in PICU got less than $60 \%$ of their prescribed energy intake; this is consistent with the reported studies in critically ill children of energy delivery ranging from 37 to $60 \%$ of the child's predicted requirements ${ }^{(5,6,12,13)}$.

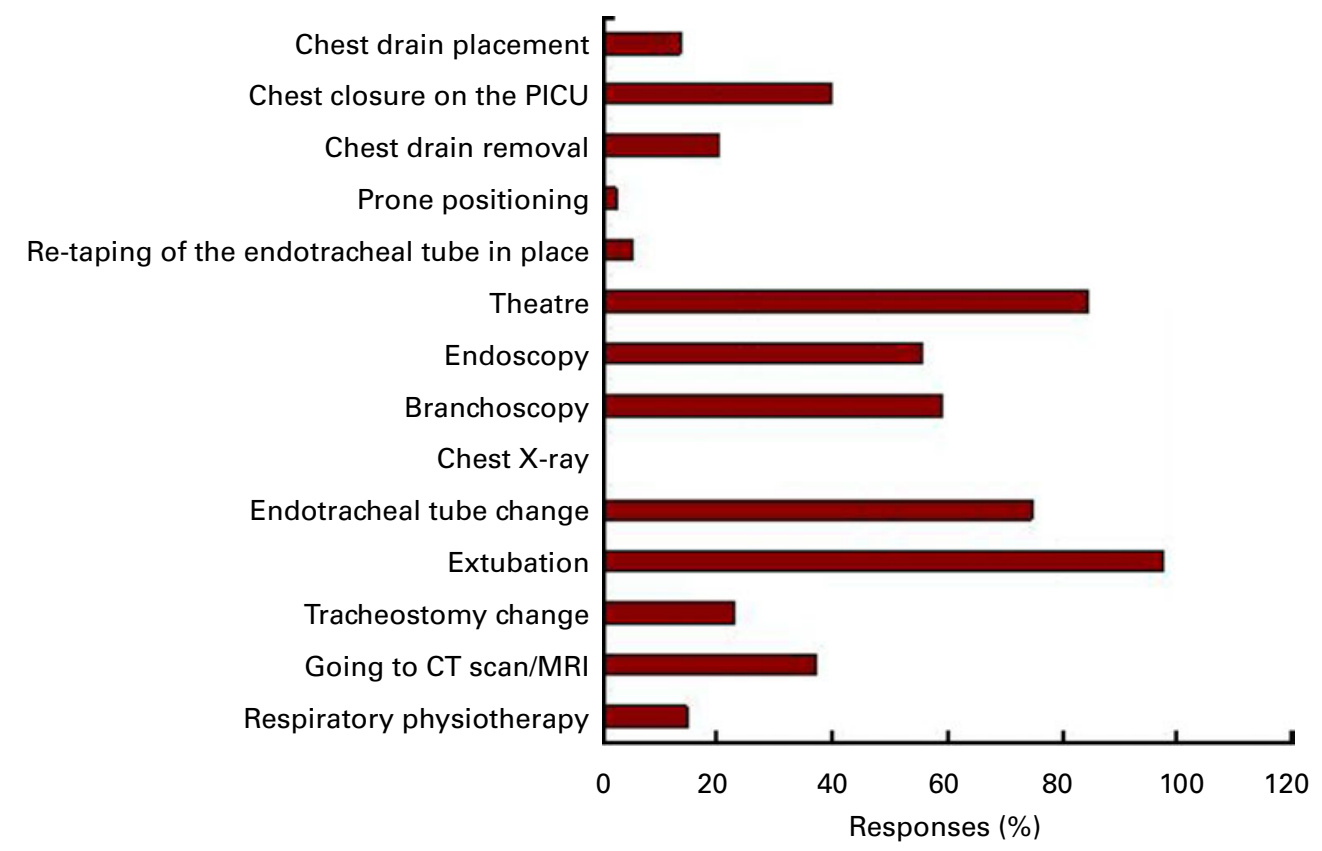

Fig. 3. Pediatric intensive care unit (PICU) procedures that patients were fasted for, in response to the question 'For an average intubated and naso-gastrically fed child on the PICU which of these procedures would you fast the child before? Please tick all that apply'. CT, Computerised tomography; MRI, magnetic resonance imaging. (A colour version of this figure can be found online at http://www.journals.cambridge.org/bjn). 


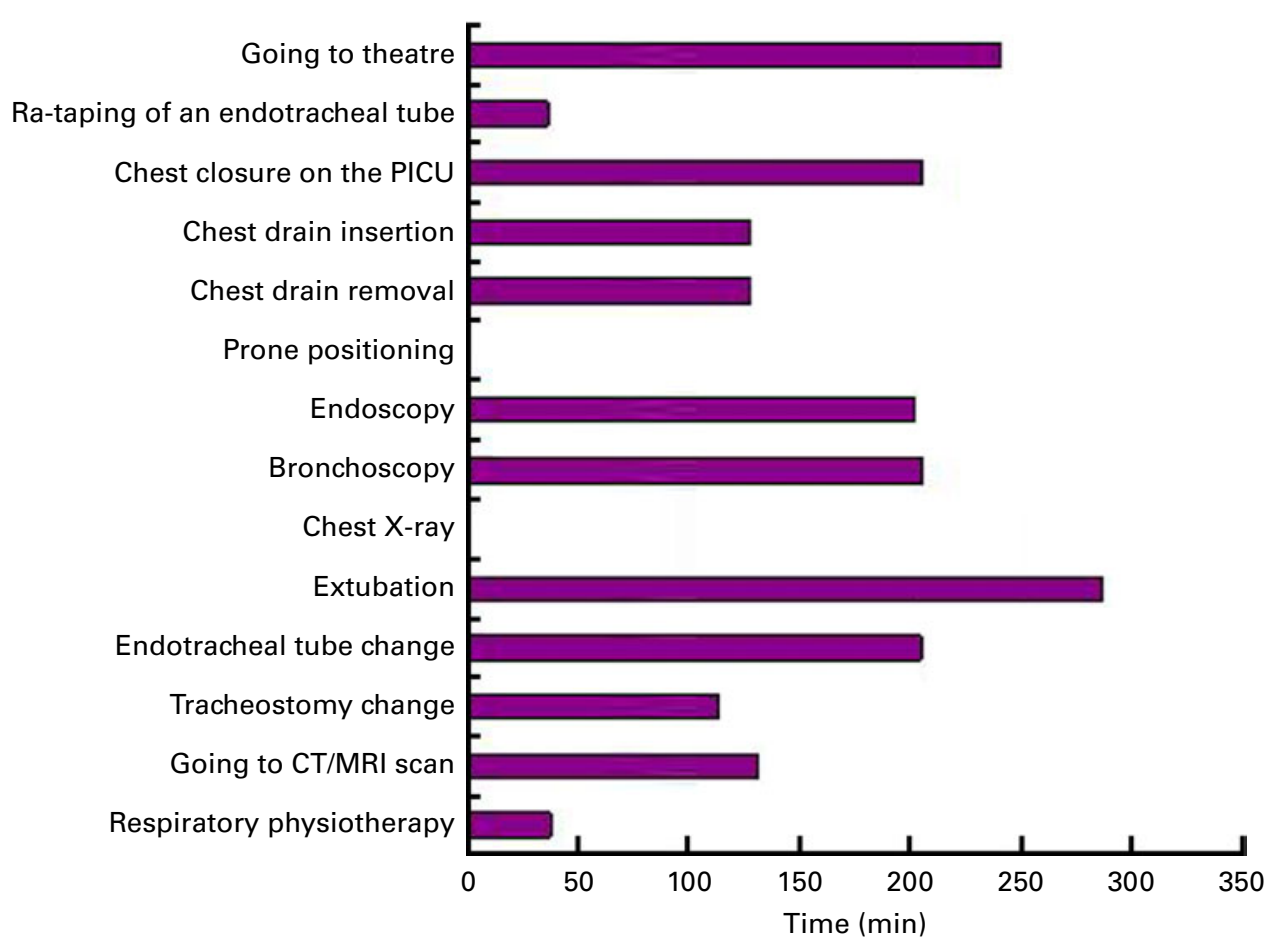

Fig. 4. Mean total fasting (before and after), in response to the question 'For the procedures you indicated previously that you would fast the child for how long would they be fasted for? (minutes in total before and after the procedure)'. PICU, paediatric intensive care unit; CT, computerised tomography; MRI, magnetic resonance imaging. (A colour version of this figure can be found online at http://www.journals.cambridge.org/bjn).

In terms of absolute contraindications to EN in PIC, suspected NEC was the most prominent with immediate bowel rest as a key treatment strategy in the management of suspected $\mathrm{NEC}^{(14)}$. Although $12 \%$ of respondents' proposed high serum lactates as an absolute contraindication, PIC evidence does not support this. However, in adult patients ( $n$ 128), a high admission serum lactate was highly predictive of gastrointestinal dysfunction ${ }^{(15)}$. Coarctation of the aorta reduces systemic and mesenteric blood flow pre-operatively and mesenteric blood flow is also altered post-operatively ${ }^{(16)}$. Although $12 \%$ of respondents stated coarctation of the aorta as a contraindication, there is only one published case of NEC in a neonate where coarctation of the aorta was identified $^{(17)}$. Most of these perceived contraindications seem to be based on risk aversion strategies.

In relation to the initiation of enteral feeds, although $45 \%$ of respondents had no specific target start time to start EN, $24 \%$ stated they would start within $4-6 \mathrm{~h}$ of PICU admission. A systematic review of early enteral feeding $(<36 \mathrm{~h}$ after ICU admission) compared to late in critically ill adults showed that early enteral feeding was associated with significantly lower incidence of infections $(P \leq 0.0006)$ and a reduced length of hospital stay $(P=0 \cdot 004)^{(18)}$. Another meta-analysis demonstrated that even earlier enteral feeding $(<24 \mathrm{~h}$ of ICU admission) reduced mortality in critically ill adults ${ }^{(19)}$. Others have showed that early ( $<6 \mathrm{~h}$ after ICU admission) EN was possible and improved time to achieve energy goal; however, there is no evidence of the effect of early EN on outcomes in children ${ }^{(11)}$.

The concept of trophic feeding originated in feeding preterm infants and has demonstrated some benefits in them ${ }^{(20-22)}$. However, a Cochrane review could not recommend this practice ${ }^{(23)}$. Despite the uncertainty about whether benefits seen in preterm neonates can be extrapolated to critically ill children, trophic feeding is widespread in PICU in the UK and Ireland. A total of $86 \%$ of the respondents used trophic feeds. However, what was considered trophic varied considerably. The concept of early 'trickle' or 'trophic' feeds was recommended in the American Society for parenteral and Enteral Nutrition (ASPEN) adult nutrition guidelines ${ }^{(24)}$ and tested in a randomised controlled trial in ventilated adults with respiratory failure ${ }^{(25)}$. Limited evidence supports the theory and practicalities of trophic feed administration.

The GRV featured prominently in the decision to both stop EN and to determine feed tolerance, and was similar for all professional groups; however, evidence to support the use of GRV is problematic. The risk of potential aspiration of gastric contents is reported as high in critically ill patients and a major risk factor for the development of pneumonia ${ }^{(26)}$. However, this level of risk is difficult to quantify. Multiple factors can affect the gastric emptying rate and thus an increase in GRV in PIC children ${ }^{(27-29)}$. The direct measurement of gastric emptying is difficult in clinical practice; only one PICU in the UK measures gastric emptying time on all children. Additionally, the correlation between GRV and gastric emptying remains unclear ${ }^{(29)}$. The present results show both a large variation in what is considered an acceptable GRV and the variations in how GRV is measured across PICU. Given that, in PICU, the majority of children are aged $<12$ months, then it is unsurprising that uncertainty increases when considering larger children and adolescents. Considerable debate occurs about the utility of GRV measurement in critically ill patients 
receiving EN. An adult study found that early EN without GRV monitoring improved the delivery of EN and did not increase vomiting or ventilator-associated pneumonia ${ }^{(30)}$. These findings are supported by a further randomised controlled trial of 328 adults that found that a GRV of $500 \mathrm{ml}$ was not associated with any adverse events or gastrointestinal complications $^{(31)}$. In measuring GRV, there is a presumption that the measured volume is accurate. However, various factors affect this, including the syringe size, the type and lumen size of the feeding tube, position of the patient and the feeding tube in the stomach and aspiration technique ${ }^{(27,32)}$. Small lumen tubes, small size aspirating syringes and collapsible soft feeding tubes can all produce falsely low GRV, as can adherence of the tip of the tube to the gastric mucosa or positioning within the stomach where gastric fluid has not accumulated $^{(27,32)}$. The respondents appeared to be unaware of some of these factors when making decisions about the GRV. Technical factors affecting GRV (e.g. equipment used and nurse's technique) were rated less important in affecting the amount of aspirate obtained - implying a reduced awareness of these factors. For both patient and technical factors, nurses played significantly more importance on the site of the feeding tube, perhaps reflecting their core responsibility in confirming the tube placement.

Stopping feeds for a while and rechecking aspirate was the first course of action that respondents proposed, followed by changing the continuous feeds and then starting a pro-kinetic agent. Although the adult ASPEN guidelines recommend the use of pro-kinetic agents ${ }^{(24)}$, a systematic review in neonates was unable to make any recommendations, and the present ASPEN paediatric guidelines do not recommend their use ${ }^{(4)}$. Despite this, $82 \%$ of respondents said they sometimes or always used pro-kinetic agents. Significantly more nurses would consider changing to continuous feeds $(P=0 \cdot 019)$ if the child was not tolerating bolus feeds. Furthermore, just over one-half of our respondents said their default method to start EN was continuous nasogastric feeds. No studies have ever shown continuous feeds to be superior to intermittent feeds in terms of GRV. Only one randomised controlled trial ( $n$ 45) has examined continuous $v$. bolus feeds in PICU patients, and this showed no differences in GRV between the two methods ${ }^{(33)}$. Studies in preterm infants also found no difference in GRV, although they showed a higher GRV in the continuously fed group ${ }^{(34-37)}$

The critical care literature (adult, child and neonatal) frequently cites feed interruptions as a major problem for reducing energy delivery in $\mathrm{ICU}^{(38)}$. A small study revealed that feed interruptions for procedures requiring fasting occurred in $43 \%$ children in one $24-\mathrm{h}$ period (mean fasting time was $8.9 \mathrm{~h})^{(6)}$. This is consistent with other paediatric studies ${ }^{(5)}$ and adult ICU studies ${ }^{(39,40)}$. The present study showed considerable variation across respondents for both the procedures that children were fasted for and the mean fasting time. Critically ill children are likely to have delayed gastric emptying, and the mean fasting time in the present survey before extubation or anaesthesia was $6 \mathrm{~h}$, which seems reasonable. Although neither the procedures fasted for nor the duration of fasting are based on any evidence, the degree to which these are applied within a unit does significantly make an impact on the amount of enteral feed delivered and reflects a unit's risk aversion strategy.

\section{Limitations}

Because the unit response rate varied from 1 to 21 (mean 3) responses per unit, some larger units may be over-represented in the results. In addition, our technique of secondary invitation of respondents by selected lead individuals within a unit could introduce selection bias and we acknowledge this; however, guidance was provided to them to circulate to a mix of professionals with varying degrees of experience and education. There was a predominance of nursing respondents (59\%) and, although this arguably over-represents one disciplinary perspective, it does reflect the reality of staff mix in PICU. Again, because of our design, we do not know details about non-responders, and this again may introduce bias in our sample. The small numbers of non-PIC-trained staff (both doctors and nurses) did not allow the comparison between PIC education of staff and responses, and the small cell frequencies in some analyses was a limitation when undertaking the $\chi^{2}$ test. Given the issue of GRV, in retrospect, perhaps we could have asked whether GRV was discarded or returned, and it would also have been interesting to ask about the use of post-pyloric feeding, which we did not. The large number of free comments provided by respondents made quantitative analysis challenging, but reflects the PIC experience of these clinicians, the variability of patient conditions and ages and gives insight into the range of views. However, the survey's strengths are its multi-disciplinarity and the $90 \%$ response rate from across the UK and Ireland.

\section{Recommendations for practice and research}

Practice recommendations include improving the standardisation and consistency for EN. Agreed fasting times for regular interventional procedures on PICU would improve feeding times, as would an agreed method of measuring GRV and defining an acceptable volume. The results of this survey clearly illuminate the diversity and uncertainty about the management of EN in PIC. The authors propose that the present study provides a robust first step in identifying the core areas of concern and inconsistency and that the development of national consensus guidelines for EN in PIC is warranted. Such guidelines would need to be built upon both robust evidence and the expert consensus from across the UK with the potential for the guidelines to use a traffic light system (red - contraindicated, orange - unsure, green - acceptable) to assist in decision making. Enhanced education about factors affecting GRV should be provided to the PIC staff. A national multi-disciplinary PIC EN research and advisory group should be established to promote more collaborative research and improve nutrition in PIC across the UK and Ireland. In terms of research priorities, the most accurate method for the measurement of GRV, determining the risk of aspiration in critically ill children, its relationship to GRV and whether this is this the same across all age profiles are key priorities. 
Other priorities include evidence for measuring gastric emptying time in critically ill children and whether this affects nutritional outcomes (feed tolerance and energy delivery) and the role of the dietitian in clinical feeding problems in the PICU.

\section{Conclusions}

The present survey has highlighted the variability of the present enteral feeding practices across the UK and Ireland, particularly with regard to the use of GRV and fasting for procedures. These findings are similar to other published work internationally in terms of practice variations, but highlight a number of recommendations for both practice and research, which the PIC and dietetic communities should act on.

\section{Acknowledgements}

The authors wish to thank all the PICU staff in the UK and Ireland who participated in the present survey and PICS-SG for supporting this project and $\mathrm{Mr}$ Andy Darbyshire for his involvement in this project. The author's contributions are as follows: L. T., B. C., L. L. designed the study; L. T. collected and analysed the data; L. T., B. C., L. L. wrote the manuscript. The authors declare no conflict of interest.

\section{References}

1. Irving S, Simone S, Hicks F, et al. (2000) Nutrition for the critically ill child: enteral and parenteral support. AACN Clin Issues 11, 541-558.

2. Briassoulis G, Zavras N \& Hatzis T (2001) Effectiveness and safety of a protocol of early intragastric feeding in critically ill children. Ped Crit Care Med 2, 113-121.

3. Simpson F \& Doig G (2005) Parenteral versus enteral nutrition in the critically ill patient: a meta-analysis of trials using the intention to treat principle. Intensive Care Med 31, $12-23$.

4. Mehta N, McAleer D, Hamilton S, et al. (2010) Challenges to enteral nutrition in a busy multidisciplinary PICU. JPEN 34 , $38-45$.

5. Rogers E, Gilbertson H, Heine R, et al. (2003) Barriers to adequate nutrition in critically ill children. Nutrition $\mathbf{1 9}$, $865-868$

6. Tume L, Latten L \& Darbyshire A (2010) An evaluation of enteral feeding practices in critically ill children. Nurs Crit Care 15, 291-299.

7. Mateo M (1996) Nursing management of enteral tube feedings. Heart Lung 25, 318-323.

8. Marshall A \& West S (2006) Enteral feeding in the critically ill: are nursing practices contributing to hypocaloric feeding? Intensive Crit Care Nurs 22, 1-5.

9. Fulbrook P, Bongers A \& Albarran J (2007) A European survey of enteral nutrition practices and procedures in adult intensive care units. J Clin Nurs 16, 2132-2214.

10. Hill S, Nielsen M \& Lennard-Jones J (1995) Nutritional support in intensive care units in England and Wales: a survey. Eur J Clin Nutr 49, 371-378.

11. Petrillo-Albarano T, Pettignano R, Asfaw M, et al. (2006) Use of a feeding protocol to improve nutritional support through early, aggressive enteral nutrition in the paediatric intensive care unit. Pediatric Crit Care Med 7, 340-344.
12. deNeef M, Geukers V, Dral A, et al. (2008) Nutritional goals, prescription and delivery in a pediatric intensive care unit. Clin Nutr 27, 65-71.

13. Taylor R, Preedy V, Baker A, et al. (2003) Nutritional support in critically ill children. Clin Nutr 22, 365-369.

14. Lee J \& Polin R (2003) Treatment and prevention of necrotizing enterocolitis. Sem Neonatol 8, 449-459.

15. Cresci G \& Cue J (2008) The Patient with circulatory shock: to feed or not to feed? Nutr Clin Prac 23, 501-509.

16. Ho E \& Moss A (1972) The syndrome of "mesenteric arteritis" following surgical repair of aortic coarctation. Report of nine cases and review of the literature. Pediatrics 49 , $40-45$.

17. Hasegawa T, Yoshioka Y, Sasaki T, et al. (1997) Necrotizing enterocolitis in a term infant with coarctation of the aorta complex. Ped Surg 12, 57-58.

18. Marik P \& Zaloga G (2001) Early enteral nutrition in acutely ill patients: a systematic review. Crit Care Med 29, 2264-2270.

19. Doig G, Heighes P, Simpson F, et al. (2009) Early nutrition, provided within $24 \mathrm{~h}$ of injury or intensive care unit admission, significantly reduces mortality in critically ill patients: a meta-analysis of randomised controlled trials. Int Care Med 35, 2018-2027.

20. Burrin D, Stoll B, Jiang R, et al. (2000) Minimal enteral nutrient requirements for intestinal growth in neonatal piglets: how much is enough? Am J Clin Nutr 71, 1603-1610.

21. McClure R \& Newell S (1999) Randomised controlled trial of trophic feeding and gut motility. Arch Dis Child Neonatal Ed 80, F54-F58.

22. McClure R \& Newell S (2000) Randomised controlled study of clinical outcome following trophic feeding. Arch Dis Child Neonatal Ed 82, F29-F33.

23. Bombell S \& McGuire W (2009) Early trophic feeding for very low birth weight infants. The Cochrane Database of Systematic Reviews issue 3, CD000504.

24. McClave S, Martindale R, Vanek V, et al. (2009) Guidelines for the provision and assessment of nutrition support therapy in the adult critically ill patient. JPEN 33, 277-316.

25. Rice T, Mogan S, Hays M, et al. (2011) Randomized trial of initial trophic versus full-energy enteral nutrition in mechanically ventilated patients with acute respiratory failure. Crit Care Med 39, 967-974.

26. Metheny N, Clouse R, Chang Y, et al. (2006) Tracheobronchial aspiration of gastric contents in critically ill tube-fed patients: frequency, outcomes and risk factors. Crit Care Med 34, 1007-1015.

27. Gonzalez J (2008) Gastric residuals - are they important in the management of enteral nutrition? Clin Nutr High 4 , $2-7$.

28. Deane A, Chapman M, Fraser R, et al. (2007) Mechanisms underlying feed intolerance in the critically ill: implications for treatment. World J Gastroenterol 13, 3909-3917.

29. Fruhwald S, Holzer P \& Metzler H (2008) Gastrointestinal motility in acute illness. Wien Klin Wochenschr 120, 6-17.

30. Poulard F, Dimet J, Martin-Lefevre L, et al. (2010) Impact of not measuring gastric volume in mechanically ventilated patients receiving early enteral feeding: a prospective study. JPEN 34, 125-130.

31. Montejo J, Minambres E, Bordeje L, et al. (2010) Gastric residual volume during enteral nutrition in ICU patients: the REGANE study. Int Care Med 36, 1386-1393.

32. Metheny N (2008) Residual volume measurement should be retained in enteral feeding protocols. Am J Crit Care 17, 62-64. 
33. Horn D, Chaboyer W \& Schluter P (2004) Gastric residual volumes in critically ill paediatric patients: a comparison of feeding regimes. Aust Crit Care 17, 98-103.

34. Kocan M \& Hickisch S (1986) A comparison of continuous and intermittent enteral nutrition in NICU patients. J Neurosci Nurs 18, 333-337.

35. Toce S, Keenan W \& Homan S (1987) Enteral feeding in very low birth weight infants. Arch Ped Adol Med 141, 439-444.

36. Silvestre M, Marbach C, Brans Y, et al. (1996) A prospective randomised controlled trial comparing continuous versus intermittent feeding methods in very low birth weight neonates. J Pediatr 128, 748-752.
37. Schanler R, Schulman R, Lau C, et al. (1999) Feeding strategies for premature infants: randomised trial of gastrointestinal priming and tube-feeding method. Pediatrics 103, 434-439.

38. Mehta N (2009) Approach to enteral feeding in the PICU. Nutr Clin Pract 24, 337-387.

39. Morgan L, Dickerson R, Alexander K, et al. (2004) Factors causing interrupted delivery of enteral nutrition in trauma intensive care patients. Nutr Clin Pract 19, 511-517.

40. O'Meara D, Mireles-Cabodevila E, Frame F, et al. (2008) Evaluation of delivery of enteral nutrition in critically ill patients receiving mechanical ventilation. Am J Crit Care 17, 53-61. 


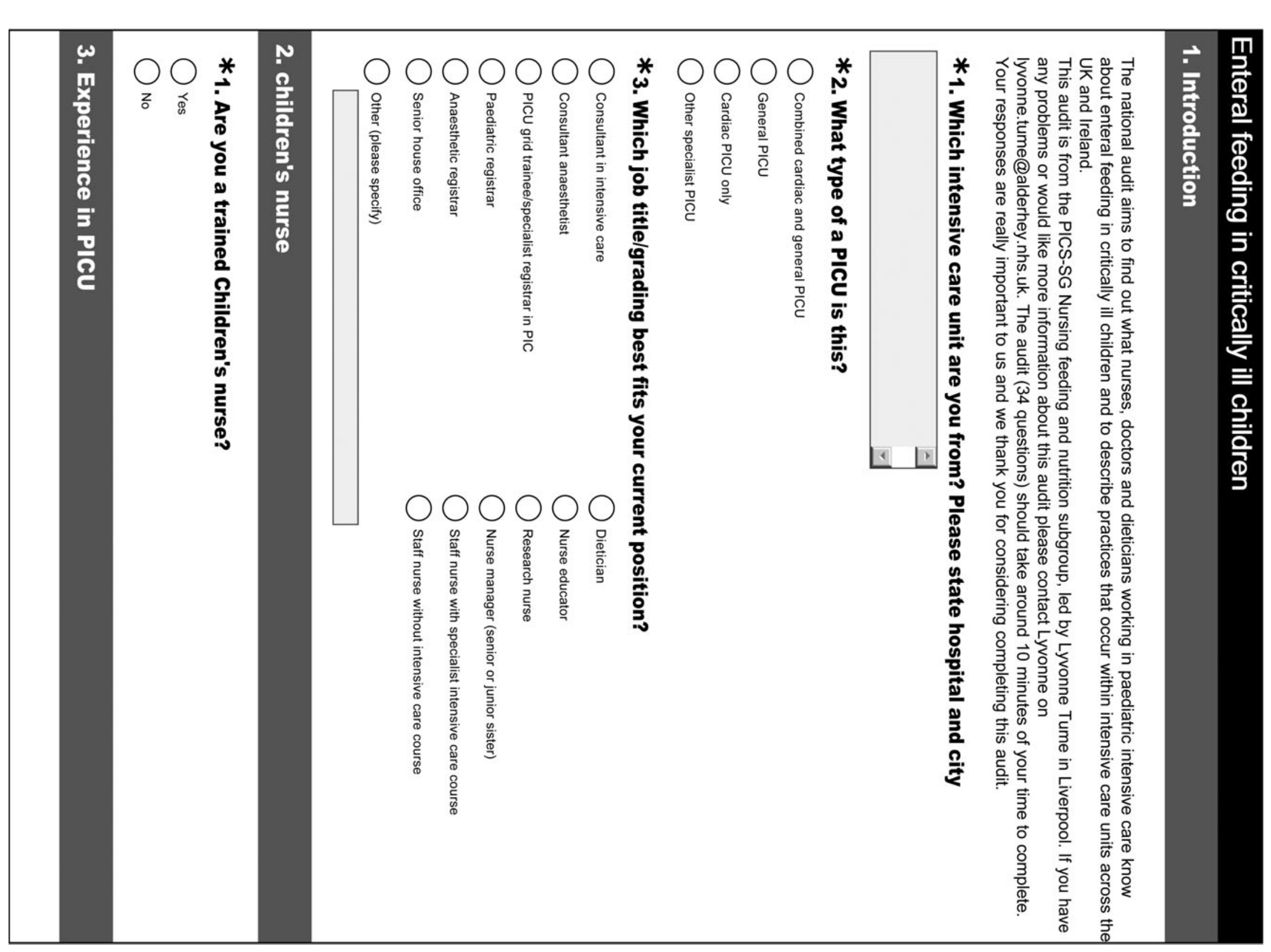

흥 


\section{Enteral feeding in critically ill children}

\section{*1. How many years experience do you have of working in PICU?}
$\bigcirc<6$ months
○ $6-<12$ months
12 months $-<2$ years

$2-<5$ years

$5-<10$ years

$\bigcirc>10$ years

\section{Nutritional information}

*1. On average (over the first three days of a child's PICU admission), how much of their prescribed calorie requirements do you think a child actually receives?
$<20 \%$
$20-<40 \%$
- $40-<60 \%$
$60-<80 \%$
$>80 \%$

*2. What do you think are the TOP three (most common) reasons why children in PICU may not get adequate calories?

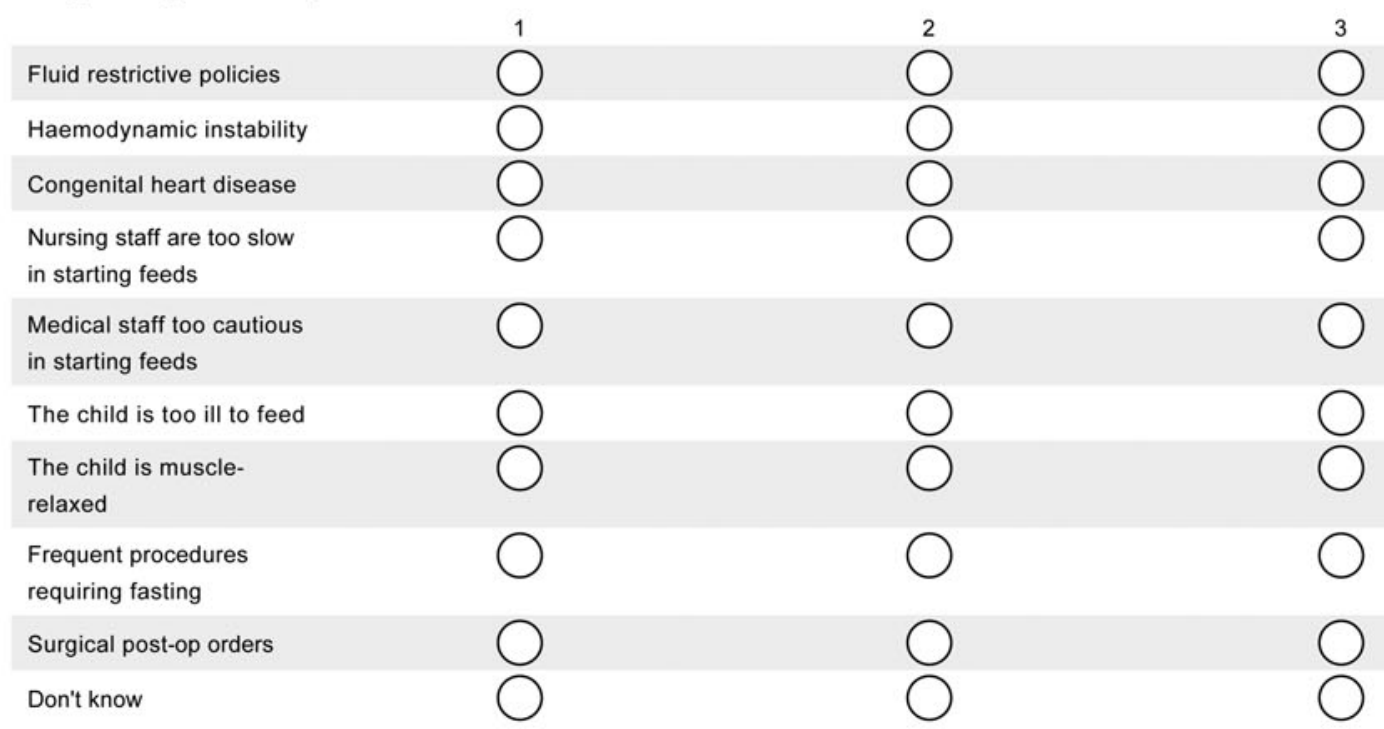

Other (please specify) 
NS British Journal of Nutrition

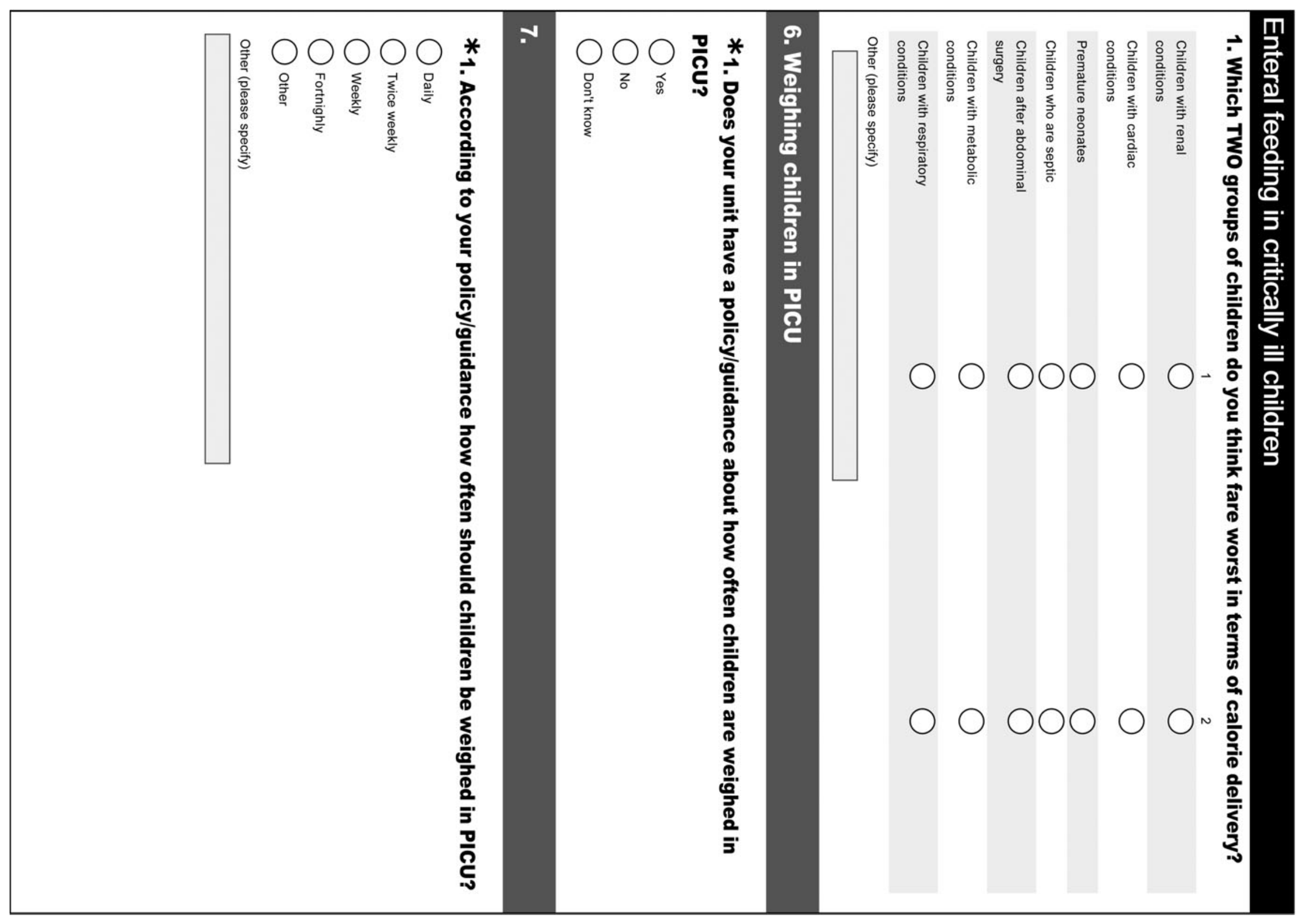




\section{Enteral feeding in critically ill children}

\section{*2. When do you think children should be weighed in PICU?}

Children who have been in PICU $>1$ week

$\bigcirc$ Only for specific indications

$\bigcirc$ Don't know

$\bigcirc$ Other

Other (please specify)

\section{Starting enteral feeds}

*1. Are there any clinical conditions that you think are absolute contraindications to enteral feeding?
Yes
No
Don't know

\section{Contraindications for enteral feeding}

*1. Which of the following conditions do you think are absolute contraindications to enteral feeding?

Severe sepsis

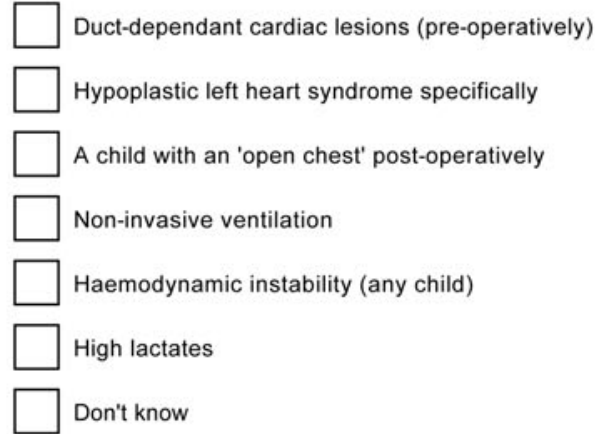

Other (please specify)

\section{The use of feeding guidelines}

*1. Do you have formal written guidelines or protocols for enteral feeding on PICU?

$\bigcirc$ Yes

$\bigcirc$ No

Don't know 


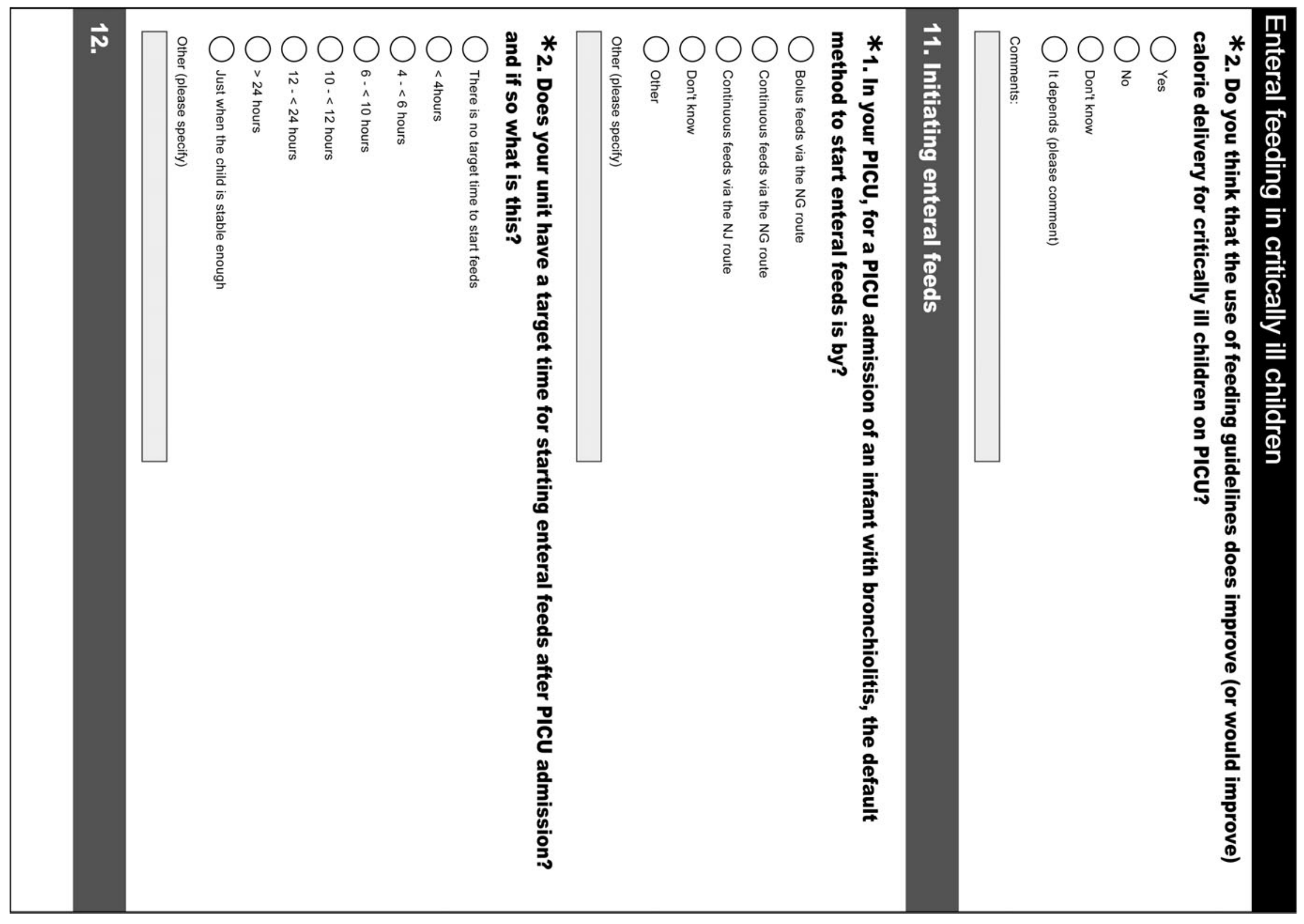




\section{Enteral feeding in critically ill children}

\section{*1. Do you think this target time is?}

Too soon

Too late

About right

Don't know

*2. If the child is very ill and cannot start full feeds, would you consider using trophic feeds?

Yes

$\bigcirc$ No

Don't know

Other

Other (please specify)

\section{3.}

*1. What amounts and frequency do you consider to be 'trophic' feeds?

\section{Stopping enteral feeds}

*1. What are TOP 3 clinical reasons you would STOP enteral feeds?

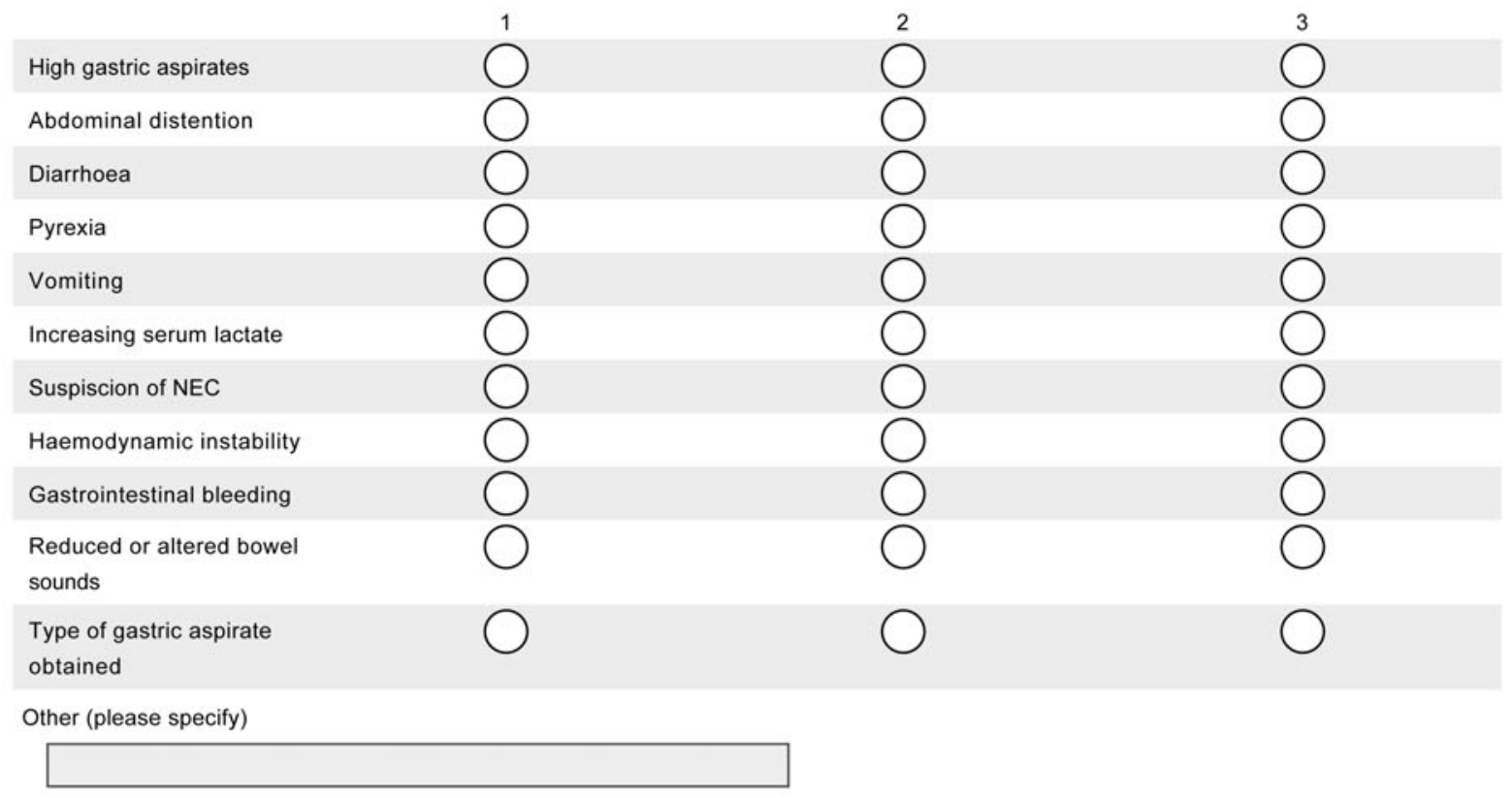


NS British Journal of Nutrition

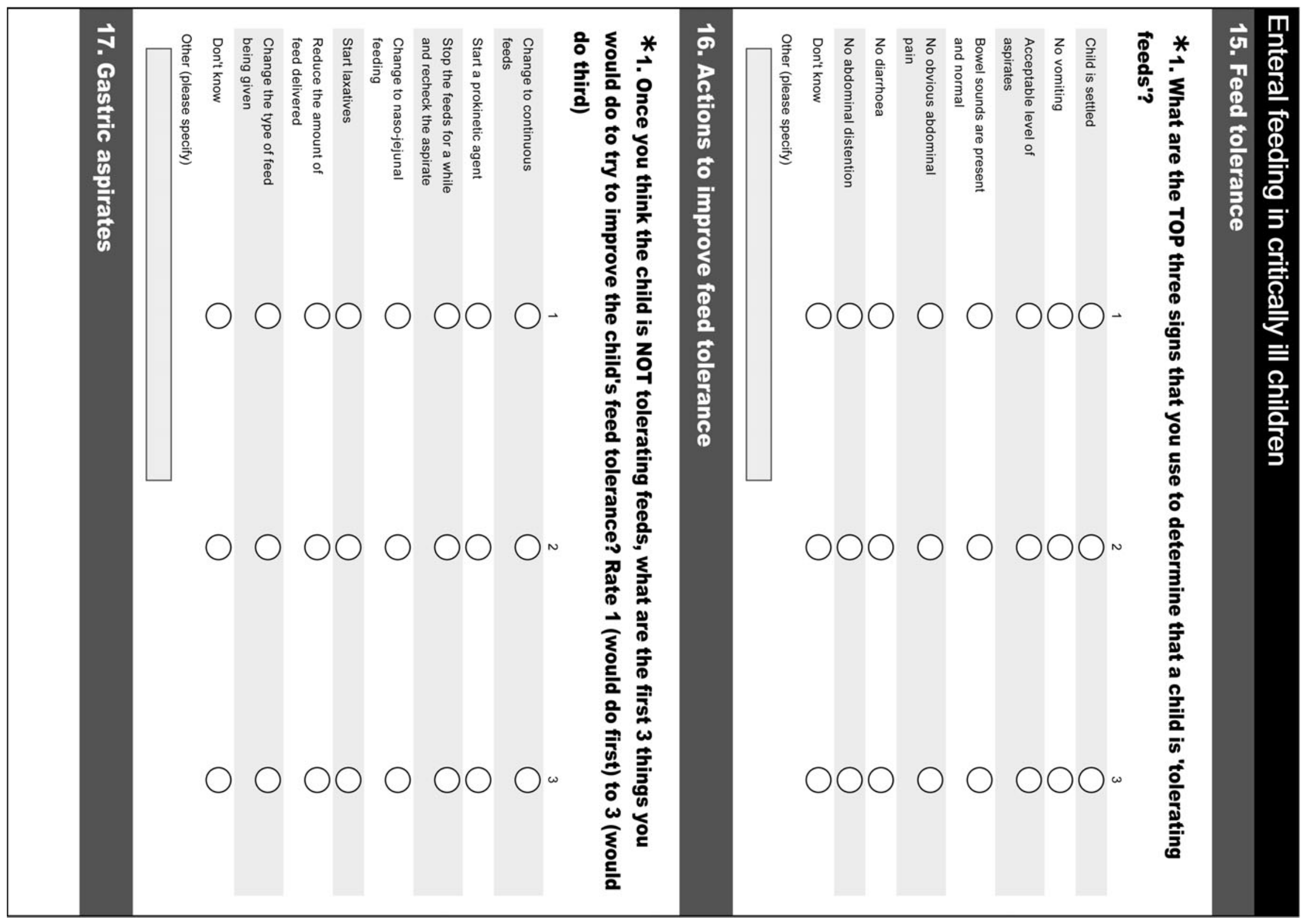




\section{Enteral feeding in critically ill children}

*1. What is the MAXIMAL gastric aspirate you would tolerate before stopping feeds on a) a $5 \mathrm{~kg}$ infant
$\bigcirc 15 \mathrm{ml}$
$\bigcirc 20 \mathrm{ml}$
$\bigcirc 25 \mathrm{ml}$
$\bigcirc 30 \mathrm{ml}$
$\bigcirc 45 \mathrm{ml}$
$\bigcirc 50 \mathrm{ml}$
$\bigcirc_{\text {know }}$ Don't
Other

Other (please specify)

*2. What is the MAXIMAL gastric aspirate you would tolerate before stopping feeds on b) a $65 \mathrm{Kg}$ adolescent
$\bigcirc 100 \mathrm{ml}$
$150 \mathrm{ml}$
$200 \mathrm{ml}$
250ml
$300 \mathrm{ml}$
$350 \mathrm{ml}$
○ $400 \mathrm{ml}$
$\bigcirc_{\text {know }}$ Don't $\bigcirc$ other

Other (please specify)

\section{Patient related factors}

*1. What are the TOP three (3) patient related factors do you think might affect the amount of gastric aspirate you get back? Rate from 1 (most important) to 3 (less important)

Type of feed
Site of feed eg NG, NJ or
gastrostomy
Patient's weight
The use of continuous
feeds
The volume of gastric and
salivary juice produced by
patient
Don't know

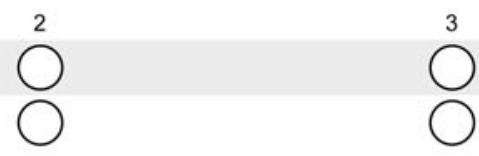

Other (please specify)

\section{Technical factors relating to enteral feeding}


NS British Journal of Nutrition

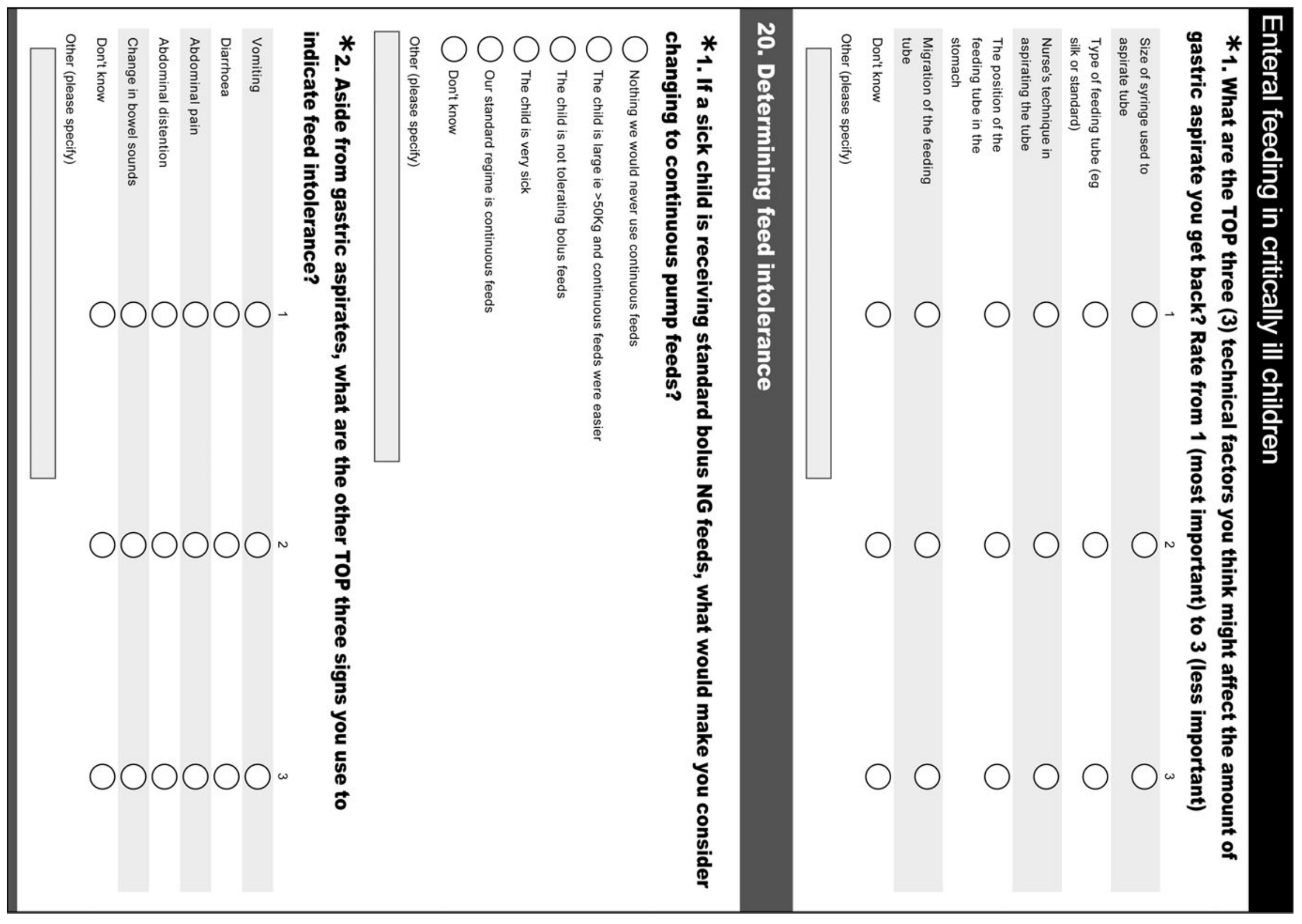




\section{Enteral feeding in critically ill children \\ 21. Improving feed tolerance}

*1. Do you think that feed intolerance could be an early sign of necrotising enterocolitis?

$\bigcirc$ Yes

$\bigcirc$ No

Don't know

*2. Are prokentics used in your PICU to improve feed intolerance?

Yes always

Yes sometimes

Rarely

$\bigcirc$ Never

Don't know

Other (please specify)

*3. If a child was persistently not tolerating enteral feeds, at what point would you consider starting parenteral nutrition?

<24 hours
$24-<48$ hours
$48-<72$ hours
Other (please specify)

$>72$ hours

Don't know

\section{Fasting for procedures}

*1. For an average intubated and naso-gastrically fed child on the PICU which of these procedures would you fast the child before? Please tick all that apply

Respiratory physiotherapy

$\square$ Endoscopy

Going to CT scan/MRI

$\square$ Theatre

$\square$ Tracheostomy change

Re-taping of the endotracheal tube in place

Extubation

Prone positioning

Endotracheal tube change

Chest drain removal

Chest x-ray

Chest closure on the PICU

Bronchoscopy

Chest drain placement 


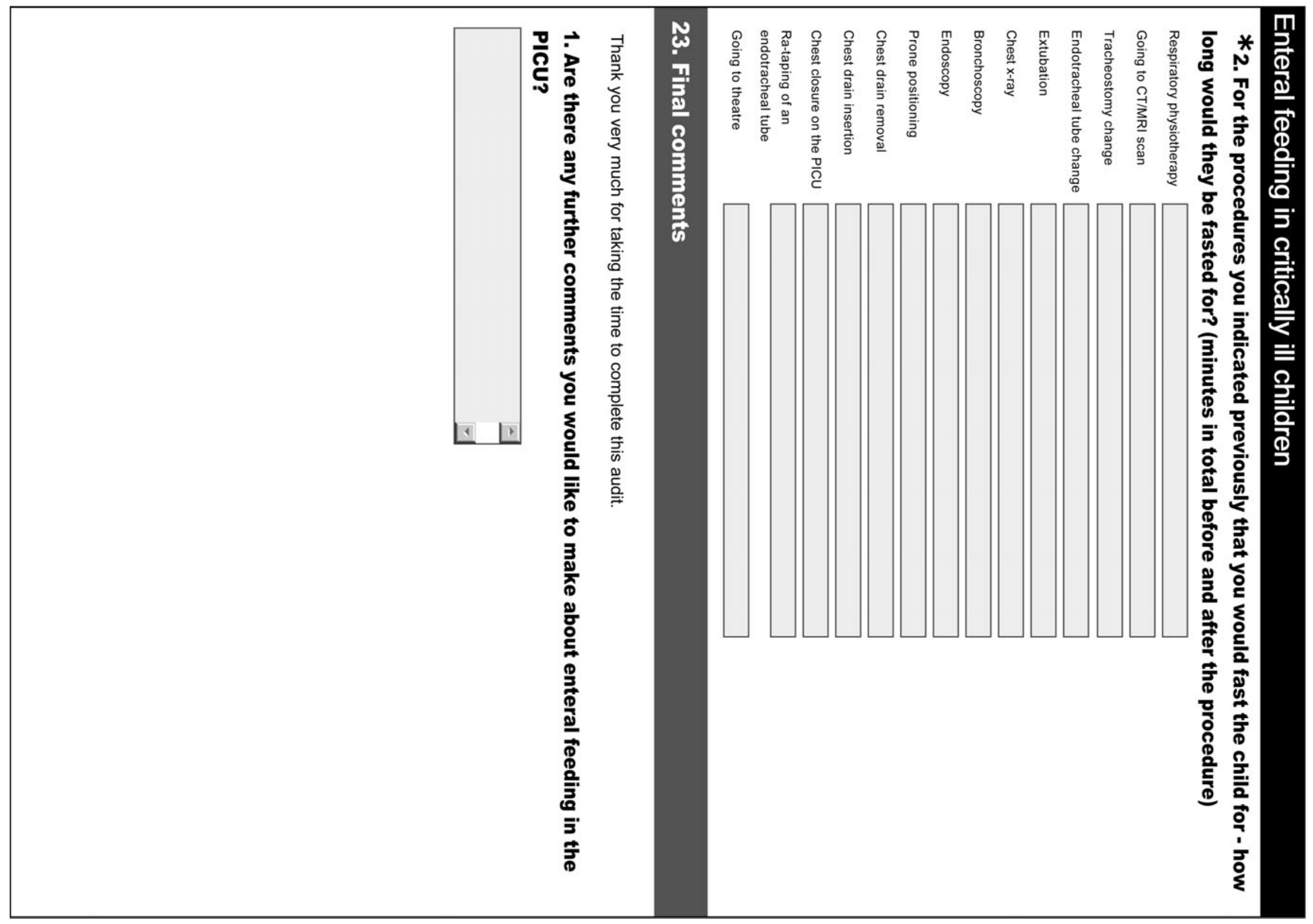

